\title{
The Boltzmann Equation for Weakly Inhomogeneous Data
}

\author{
L. Arkeryd ${ }^{1}$, R. Esposito ${ }^{2 \star}$ and M. Pulvirenti ${ }^{2 \star}$ \\ 1 Department of Mathematics Chalmers University of Technology and the University of Goteborg, \\ Sweden \\ 2 Dipartimento di Matematica Universita, "La Sapienza" di Roma, Italy.
}

\begin{abstract}
We solve the initial value problem associated to the nonlinear Boltzmann equation in the case in which the initial distribution has sufficiently small spatial gradients.
\end{abstract}

\section{Introduction and Notation}

The nonlinear Boltzmann equation is believed to describe the time evolution of a rarefied gas of particles. It takes the form

$$
D_{t} f:=\left(\partial_{t}+v \cdot \nabla_{x}\right) f=Q(f, f), \quad f(0)=f_{0},
$$

where $D \times \mathbb{R}^{3} \ni(x, v) \rightarrow f(x, v)$ is the distribution of the particles, $x$ and $v$ denote position and velocity respectively, $D \subset \mathbb{R}^{3}$ is the domain to which the gas is confined. The bilinear operator $Q$ takes into account the interaction among the particles and will be specified later.

The initial value problem (1.1) has been considered by several authors, and the following three groups of results global in time are available;

a) Spatially Homogeneous case. If $f_{0}$ depends only on $v, f_{t}$ has still this property. In this case global existence and uniqueness result can be proved for very general data.

b) Small Deviations from Equilibrium. It is well known that the nontrivial equilibria for the problem (1.1) are the Maxwellian distributions. An initial distribution $f_{0}$, slightly differing from a Maxwellian, can be proved to evolve globally and uniquely in time, according to Eq. (1.1). Moreover it approaches a Maxwellian asymptotically in time.

c) Small Deviations from Vacuum. A global existence and uniqueness theorem for the initial value problem (1.1) can be proved for an initial distribution $f_{0}(x, v), x \in \mathbb{R}^{3}$,

* Supported by C.N.R.-GNFM and MPI 
decaying for large values of $|x|$ and $|v|$ and under suitable smallness assumptions on $f_{0}$.

The first results in the above directions were obtained by Carleman [1], Ukai [2] (following a previous analysis due to Grad [3]) and Illner and Shinbrot [4] respectively.

Finally it should be noticed that a global existence theorem for the initial value problem (1.1), also for large data, has been proved in [5] in the framework of the Loeb integration. However this result does not clarify whether the solutions to the Boltzmann equation (for more general data than those of the kind a), b), c) above) develop singularities in finite times or not.

In the present paper we construct a unique solution to the Boltzmann problem (1.1) for initial distributions near, in a suitable sense, to an homogeneous datum. Namely, let us consider the gas in a three dimensional flat torus $T=[0,1]^{3}$. Let $f_{0}$ be the initial distribution and

$$
g_{0}(v)=\int_{T} d x f_{0}(x, v), \quad u_{0}(x, v)=f_{0}(x, v)-g_{0}(v) .
$$

One can hope to construct solutions along the following steps.

Step 1 (Local Theorem). The evolution equation for the perturbation $u_{t}$ is bilinear. So it is natural to expect that, for any fixed arbitrary time $t$, one should be able to evolve $u_{0}$ up to $t$, provided that $u_{0}$ is sufficiently small. Moreover $u_{t}$ ought to be small together with $u_{0}$.

Step 2 (Approach to Equilibrium for the Homogeneous Equation). Let $g_{t}$ be the solution of the homogeneous equation with initial datum $g_{0}$. It is known that $g_{t}$ approaches a Maxwellian equilibrium when $t \rightarrow \infty$. Choose $t$ so large (and accordingly $u_{0}$ so small) that $g_{t}$ is arbitrarily near to a Maxwellian $\omega$.

Step 3 (Perturbation of the Equilibrium). At the time $t, f_{t}$ is near to $g_{t}$, by Step 1 . Therefore it is also near to $\omega$ by Step 2 . Then one can hope to apply the result $b$ ) to extend the solution up to time $t=\infty$.

Unfortunately the above program cannot be performed so simply just suitably combining known results. The reason is that the approach to equilibrium for the homogeneous solution (Step 2 ) is relatively easy to prove in a norm (see $\|\cdot\|_{s}$ below) in which Steps 1 and 3 become more difficult. In particular $f_{t}-\omega$, even if small in this norm, does not decay exponentially in the velocity space, and this makes the techniques of Ukai and Grad [2 and 3] not directly applicable since they make essential use of the exponential decay of the perturbations. However, using some of Carleman's techniques [1], we obtain new estimates on the collision operator (see Sect. 3), which allow us to extend the analysis of [2] to the case of perturbations only polynomially decaying (Step 3) as well as to prove the local theorem (Step 1).

We remark that the above results have an intrinsic interest besides the purposes of this paper.

After this preparation the proof of the main theorem follows easily. That is the content of Sect. 4. As for Sect. 2, it is devoted to some known properties of the homogeneous solutions which will be useful in the sequel including a result about the approach to equilibrium. 
We conclude this Section by establishing hypotheses, notation and our main theorem.

We define the (unsymmetrized) collision operator as

$$
\begin{aligned}
Q(f, g)(v) & =\int_{\mathbb{R}^{3}} d v_{1} \int_{0}^{2 \pi} d \varphi \int_{0}^{\pi / 2} d \theta\left\{f\left(v^{\prime}\right) g\left(v_{1}^{\prime}\right)-f(v) g\left(v_{1}\right)\right\} S(\theta,|V|) \\
& =J(f, g)(v)-f R g(v),
\end{aligned}
$$

where $(1.3)_{2}$ defines the gain and loss term respectively. Here $V$ denotes the relative velocity $V=v_{1}-v$ and $v^{\prime}, v_{1}^{\prime}$ are the outgoing velocities after a collision with ingoing velocities $v$ and $v_{1}$ and impact parameter $n=n(\theta, \varphi)=-\sin \theta \cos \varphi e_{1}-\sin \theta \sin \varphi e_{2}$ $+(V /|V|) \cos \theta$, where $\left(e_{1}, e_{2}, V /|V|\right)$ are an orthonormal system.

Explicitly

$$
v^{\prime}=v+(V \cdot n) n, \quad v_{1}^{\prime}=v_{1}-(V \cdot n) n .
$$

For $S$ we assume the following form:

$$
S(\theta,|V|)=h(\theta)|V|^{\beta}, \quad C_{0} \sin \theta \cos \theta \leqq h(\theta) \leqq C_{0}^{\prime} \sin \theta \cos \theta
$$

with $0<\beta \leqq 1$, so including the hard sphere case and the $k^{- \text {th }}$ power forces with $k>5$ and angular cut-off [6].

For $f: \mathbb{R}^{3} \rightarrow \mathbb{R}$ continuous, we introduce the following norm for $s>1$ :

$$
\|f\|_{s}=\sup _{v \in \mathbb{R}^{3}}\left(1+v^{2}\right)^{s}|f(v)|
$$

and for $L^{2}$-functions $f: T \rightarrow \mathbb{R}$ we define

$$
|f|_{l}^{2}=\sum_{k \in 2 \pi \mathbb{Z}^{3}}\left(1+k^{2}\right)^{l}|\hat{f}(k)|^{2}
$$

where $\hat{f}(k)$ denotes the Fourier transform of $f$.

Finally for measurable functions $f: T \times \mathbb{R}^{3} \rightarrow \mathbb{R}$, with $|f(\cdot, v)|_{l}$ continuous in $v$, we define

$$
\|f\|_{l, s}=\sup _{n v \in \mathbb{R}^{3}}\left(1+v^{2}\right)^{s}|f(., v)|_{l}
$$

and denote by $B_{s}$ and $B_{l, s}$ the Banach spaces associated to the norm (1.6) and (1.8).

Now we can state our main result.

Theorem 1. Given $\rho, \sigma>0$, consider the class of positive continuous functions $f_{0}$ on $T \times \mathbb{R}^{3}$ with $\int f_{0}(v) d v d x=\rho, \int v^{2} f_{0}(v) d v d x=\sigma$. There exist $s_{0}, l_{0}$ such that if $s>s_{0}, l>l_{0}$ and $g_{0} \in B_{s+1}$, then there is $a>0$, such that for $\left\|u_{0}\right\|_{l, s}<a$, there exists $a$ unique, positive, strong solution $f$ to the Boltzmann equation (1.1) in $B_{l-\varepsilon-1, s-(\varepsilon+1) / 2}$ for any $\varepsilon>0$. Moreover $f_{t} \in B_{l, s}, f \in C^{1}\left([0, \infty), B_{l-\varepsilon-1, s-(\varepsilon+1) / 2}\right)$, and $f_{t}$ converges to $\omega$ in $B_{l, s}$ for $t \rightarrow \infty$.

\section{Some Results on the Spatially Homogeneous Boltzmann Equation}

We need some results for the initial value problem associated to the spatially 
homogeneous Boltzmann equation:

$$
\begin{aligned}
\partial_{t} f & =J(f, f)-f R f, \\
f(0, v) & =f_{0}(v) .
\end{aligned}
$$

Proposition 2.1. There is $s_{0}>0$ such that, for all $f_{0}>0$ and $\left\|f_{0}\right\|_{s}<\infty$ for some $s>s_{0}$, there exists a unique positive solution for the problem (2.1) and a constant $C_{s}$ such that

$$
\sup _{t \geqq 0}\left\|f_{t}\right\|_{s}<C_{s}
$$

Moreover it satisfies:

$$
\begin{aligned}
& \int f(t, v) d v=\int f_{0}(v) d v, \quad \int v f(t, v) d v=\int v f_{0}(v) d v \\
& \int v^{2} f(v) d v=\int v^{2} f_{0}(v) d v, \\
& \int f(t, v) \log f(t, v) d v \leqq \int f_{0}(v) \log f_{0}(v) d v \text { for all } t>0 .
\end{aligned}
$$

Proposition 2.1 was proved by Carleman [1] for hard spheres and $s_{0}=3$. The proof for the general case is contained in [8].

Proposition 2.2. Under the conditions of Prop. 2.1 there is a $v>0$ such that

$$
R f_{t}(v)>v\left(1+|v|^{\beta}\right) \text {. }
$$

Proposition 2.2 is proved in [7] Lemma 4. The constant $v$ depends on the density and the energy of $f_{0}$ and on $C_{s}$.

Finally, we are interested in the large time behavior of the solution. Since the $\mathrm{H}$ theorem can be proved, it can be shown that the solution approaches the equilibrium for large $t$. The equilibrium corresponding to the initial datum is given by the Maxwellian with the same mass, energy and momentum as the initial datum. By a suitable choice of coordinates we can reduce it to an initial datum $f_{0}$ such that:

$$
\int f_{0} d v=1, \quad \int v f_{0} d v=0, \quad \int v^{2} f_{0} d v=3
$$

In this case

$$
\omega(v)=(2 \pi)^{-3 / 2} \exp \left(-v^{2} / 2\right)
$$

For simplicity in this paper we shall always consider initial data such that (2.5) are satisfied.

Proposition 2.3. Let $f_{0}$ be as in Proposition 2.1 and satisfy Eqs. (2.5). Then

$$
\lim _{t \rightarrow \infty}\left\|f_{t}-\omega\right\|_{r}=0
$$

for any $r<s$.

Proposition 2.3 was proved in [1] for hard spheres and then generalized to the present case in [8].

\section{Estimates of the Collision Operator}

The following useful representation of $J(f, g)$ is due to Carleman [1] p. 32. 
It is easy to verify that

$$
\left(v_{1}^{\prime}-v_{1}\right) \cdot\left(v_{2}^{\prime}-v_{1}\right)=0 \text {. }
$$

Let us denote by $E_{v_{1}, v_{1}^{\prime}}$ the plane $\left(v_{1}^{\prime}-v_{1}\right) \cdot\left(\xi-v_{1}\right)=0$. It obviously contains $v_{1}$ and is orthogonal to $v_{1}^{\prime}-v_{1}$. Then

$$
J(f, g)\left(v_{1}\right)=\int_{\mathbb{R}^{3}} d v_{1}^{\prime} f\left(v_{1}^{\prime}\right) \frac{1}{\left|v_{1}-v_{1}^{\prime}\right|^{2-\beta}} \int_{E_{v_{1} v_{1}^{\prime}}} d \xi g\left(v_{2}^{\prime}\right) G(\theta),
$$

where $d \xi$ denotes the Lebesgue measure on the plane $E_{v_{1} v_{1}^{\prime}}$ and

$$
G(\theta)=h(\theta)\left[\sin \theta(\cos \theta)^{\beta}\right]^{-1} \leqq C_{0}^{\prime}(\cos \theta)^{1-\beta} \leqq C_{0}^{\prime}
$$

Denoting by

$$
J_{0}(f, g)\left(v_{1}\right)=\int_{\mathbb{R}^{3}} d v_{2} \int_{0}^{\pi / 2} d \theta \int_{0}^{2 \pi} d \varphi f\left(v_{1}^{\prime}\right) g\left(v_{2}^{\prime}\right)\left|v_{1}-v_{2}\right|^{\beta} \sin \theta \cos \theta
$$

following Carleman [1] p. 35, one has

$$
J_{0}(f, g)=J_{0}(g, f)
$$

Therefore, for positive $f$ and $g$ :

$$
J(f, g)+J(g, f) \leqq 2 C_{0}^{\prime} J_{0}(f, g) .
$$

Proposition 3.1. Let $l>3 / 2$ and $s>4$. The following estimate holds:

$$
\left(1+v^{2}\right)^{s}|Q(f, g)|_{l}(v) \leqq C(s)\|f\|_{l, s}\|g\|_{l, s}\left(1+|v|^{\beta}\right) .
$$

Furthermore there exists a function $\varepsilon=\varepsilon(s) \rightarrow 0(s \rightarrow \infty)$, such that

$$
\begin{aligned}
\left(1+v^{2}\right)^{s}\left\{|g R f|_{l}\right. & \left.+|J(g, f)|_{l}+|J(f, g)|_{l}\right\} \\
& \leqq\|f\|_{l, \mathrm{~s}}\left\{C(s)\|g\|_{l, \mathrm{~s}+\beta / 2}+\varepsilon(s)\|g\|_{l, 3}\left(1+|v|^{\beta}\right)\right\} .
\end{aligned}
$$

Proof. For any fixed $v$ and $w, f(\cdot, v)$ and $g(\cdot, w) \in H_{l}(T)$ (Sobolev space), which is a Banach algebra for $l>3 / 2$. Then we have

$$
|f(\cdot, v) g(\cdot, w)|_{\iota} \leqq C|f(\cdot, v)|_{l}|g(\cdot, w)|_{l}
$$

therefore

$$
|J(f, g)|_{l}\left(v_{1}\right) \leqq C J_{0}\left(|f|_{l},|g|_{l}\right)\left(v_{1}\right) .
$$

To simplify the notation we denote $|f|_{l}$ and $|g|_{l}$ by $\bar{f}$ and $\bar{g}$ respectively. Furthermore, for fixed $v_{1}$ we put

$$
\chi_{i}(v)=\left\{\begin{array}{l}
1 \text { if }|v|<\left|v_{1}\right| / \sqrt{2} \\
0 \text { otherwise }
\end{array}\right.
$$

$\chi_{0}=1-\chi_{i}$ and $f_{i}, f_{0}$ will stand for $f \chi_{i}$ and $f \chi_{0}$ respectively. By the energy conservation law $J_{0}\left(f_{i}, \bar{g}_{i}\right)=0$ and hence

$$
J_{0}(\bar{f}, \bar{g})=J_{0}\left(\bar{f}_{i}, \bar{g}_{0}\right)+J_{0}\left(\bar{f}_{0}, \bar{g}_{i}\right)+J_{0}\left(\bar{f}_{0}, \bar{g}_{0}\right)
$$


We estimate separately the above three terms.

$$
\begin{aligned}
& \left(1+v_{1}^{2}\right)^{s} J_{0}\left(\bar{f}_{0}, \bar{g}_{0}\right) \leqq C\|\bar{f}\|_{s}\|\bar{g}\|_{s}\left(1+v_{1}^{2}\right)^{s} \\
& \quad \cdot \int_{\mathbb{R}^{3}} d v_{1}^{\prime} \chi_{0}\left(v_{1}^{\prime}\right) \frac{1}{\left|v_{1}-v_{1}^{\prime}\right|^{2-\beta}\left(1+v_{1}^{\prime 2}\right)^{s}} \int_{E_{v_{1} v_{1}^{\prime}}} d \xi_{\chi_{0}}\left(v_{2}^{\prime}\right) \frac{1}{\left(1+v_{2}^{\prime 2}\right)^{s}} .
\end{aligned}
$$

The last integral equals

$$
\int_{\mathbb{R}^{2} \cap\left\{P^{2}+\lambda^{2}>v_{1}^{2} / 2\right\}} \frac{d \lambda}{\left(1+P^{2}+\lambda^{2}\right)^{s}} \leqq \frac{C}{\left(1+v_{1}^{2}\right)^{s-1}},
$$

where $P$ is the distance of the origin from the plane $E_{v_{1 v^{\prime}} 1}$ and $\lambda$ is the distance of the point $v_{2}^{\prime}$ (on the plane) from the orthogonal projection of the origin on the same plane. If $s>3 / 2$ the first integral appearing in the right-handside of (3.13) is bounded by $C\left(1+\left|v_{1}\right|\right)^{-2+\beta}$ so that,

$$
\left(1+v_{1}^{2}\right)^{s} J_{0}\left(\bar{f}_{0}, \bar{g}_{0}\right) \leqq C\|\bar{f}\|_{s}\|\bar{g}\|_{s}\left(1+\left|v_{1}\right|\right)^{\beta} .
$$

Furthermore, making use of Eq. (3.14) and proceeding as in (3.13),

$$
\begin{aligned}
& \left(1+v_{1}^{2}\right)^{s} J_{0}\left(\bar{f}_{i}, \bar{g}_{0}\right) \leqq C\left(1+v_{1}^{2}\right)^{s}\|\bar{f}\|_{s}\|\bar{g}\|_{s}\left(1+v_{1}^{2}\right)^{-(s-1)} \\
& \cdot \int_{\mathbb{R}^{3}} d v_{1}^{\prime} \chi_{i}\left(v_{1}^{\prime}\right) \frac{1}{\left(1+v_{1}^{\prime 2}\right)^{s}\left|v_{1}-v_{1}^{\prime}\right|^{2-\beta}} .
\end{aligned}
$$

If $v_{1}^{\prime 2} \leqq v_{1}^{2} / 2$ we have

$$
\left|v_{1}-v_{1}^{\prime}\right| \geqq\left|v_{1}\right|-\left|v_{1}^{\prime}\right| \geqq\left(1-\sqrt{2}^{-1}\right)\left|v_{1}\right|,
$$

so that the last integral is bounded by $C\left(1+\left|v_{1}\right|\right)^{-2+\beta}$ provided that $s>3 / 2$. Finally

$$
\left(1+v_{1}^{2}\right)^{s} J_{0}\left(\bar{f}_{i}, \bar{g}_{0}\right) \leqq C\left(1+\left|v_{1}\right|^{\beta}\right)\|\bar{f}\|_{s}\|\bar{g}\|_{s} .
$$

The same estimate is obtained by means of the relation (3.5) for the term $J_{0}\left(\bar{f}_{0}, \bar{g}_{i}\right)$.

We now estimate the loss term. Since $\overline{R f} \leqq R \bar{f}$, we have:

$$
\begin{aligned}
|\bar{g} \overline{R f}|\left(1+v_{1}^{2}\right)^{s} & \leqq C\|\bar{g}\|_{s} \int d v_{2} \bar{f}\left(v_{2}\right)\left|v_{1}-v_{2}\right|^{\beta} \\
& \leqq C\|\bar{f}\|_{s}\|\bar{g}\|_{s} \int d v_{2}\left|v_{1}-v_{2}\right|^{\beta} \frac{1}{\left(1+v_{2}^{2}\right)^{s}} \\
& \leqq C\|\bar{f}\|_{s}\|\bar{g}\|_{s}\left(1+\left|v_{1}\right|^{\beta}\right),
\end{aligned}
$$

provided that $s>(3+\beta) / 2$. This achieves the proof of (3.8).

To obtain the proof of the estimate (3.8) we first remark that proceeding as above

$$
|\bar{g} \overline{R f}|\left(1+v_{1}^{2}\right)^{s} \leqq C\|\bar{f}\|_{s}\|\bar{g}\|_{s+\beta / 2} .
$$

Furthermore, using (3.6)

$$
|J(g, f)|_{l}+|J(f, g)|_{l} \leqq C J_{0}(\bar{g}, \bar{f}) \leqq C\left\{J_{0}\left(\bar{g}_{0}, \bar{f}_{i}\right)+J_{0}\left(\bar{g}_{0}, \bar{f}_{0}\right)+J_{0}\left(\bar{g}_{i}, \bar{f}_{0}\right)\right\} .
$$

For the term $J_{0}\left(\bar{g}_{0}, \bar{f}_{0}\right)$ and $J_{0}\left(\bar{g}_{0}, \bar{f}_{i}\right)$ we slightly modify (3.15) and (3.16) respectively 
to get

$$
\left(1+v_{1}^{2}\right)^{s}\left(J_{0}\left(\bar{g}_{0}, \bar{f}_{0}\right)+J_{0}\left(\bar{g}_{0}, \bar{f}_{i}\right)\right) \leqq C(s)\|\bar{f}\|_{s}\|\bar{g}\|_{s+\beta / 2}
$$

The last term in the right-handside of (3.21) requires more care. We split $J_{0}\left(\bar{g}_{i}, \bar{f}_{0}\right)$ in three terms:

$$
\begin{aligned}
& J_{0}^{1}\left(\bar{g}_{i}, \bar{f}_{0}\right)=J_{0}\left(\bar{g}_{i} \chi_{\delta}^{c}, \bar{f}_{0}\right), \\
& J_{0}^{2}\left(\bar{g}_{i}, \bar{f}_{0}\right)=J_{0}\left(\bar{g}_{i} \chi_{\delta}, \chi_{\eta} \bar{f}_{0}\right), \\
& J_{0}^{3}\left(\bar{g}_{i}, \bar{f}_{0}\right)=J_{0}\left(\bar{g}_{i} \chi_{\delta}, \bar{f}_{0} \chi_{\eta}^{c}\right),
\end{aligned}
$$

and $\chi_{\delta}, \chi_{\eta}$ are the characteristic functions of the sets $\left\{\left|v_{1}^{\prime}\right|<\delta\left|v_{1}\right|\right\}$ and $\left\{\left|v_{2}\right|<\eta\left|v_{1}\right|\right\}$ for $\delta<\eta<1$ and $\chi^{c}=1-\chi$. We notice that, fixed $v_{1}, v_{1}^{\prime}$ and a point $\xi$ in the plane $E_{v_{1} v_{1}^{\prime}}$ (and therefore fixed $v_{2}^{\prime}$ ), $v_{2}$ is determined by the collision laws so that the above definitions make sense.

We have:

$$
\begin{aligned}
& J_{0}^{1}\left(\bar{g}_{i}, \bar{f}_{0}\right)\left(v_{1}\right)\left(1+v_{1}^{2}\right)^{s} \leqq \\
& \|\bar{g}\|_{3}\|\bar{f}\|_{s}\left(1+v_{1}^{2}\right)^{s} \int_{\mathbb{R}^{3}} d v_{1}^{\prime} \chi_{i}\left(v_{1}^{\prime}\right) \chi_{\delta}^{c}\left(v_{1}^{\prime}\right) \\
& \quad \frac{1}{\left(1+v_{1}^{\prime 2}\right)^{3}\left|v_{1}-v_{1}^{\prime}\right|^{2-\beta}} \int_{E_{v_{1} v_{1}^{\prime}}} d \xi \chi_{0}\left(v_{2}^{\prime}\right) \frac{1}{\left(1+v_{2}^{\prime 2}\right)^{s}} \\
& \leqq \text { (by virtue of Eq. (3.14)). } \\
& C\|\bar{g}\|_{3}\|\bar{f}\|_{s}\left(1+v_{1}^{2}\right) \int_{\mathbb{R}^{3}} d v_{1}^{\prime} \chi_{i}\left(v_{1}^{\prime}\right) \chi_{\delta}^{c}\left(v_{1}^{\prime}\right) \frac{1}{\left(1+v_{1}^{\prime 2}\right)^{3}\left|v_{1}-v_{1}^{\prime}\right|^{2-\beta}} \\
& \leqq C\|\bar{g}\|_{3}\|\bar{f}\|_{s} \sup _{v_{1}} \frac{\left(1+v_{1}^{2}\right)}{\left(1+\delta^{2} v_{1}^{2}\right)} \int_{\mathbb{R}^{3}} d v_{1}^{\prime} \frac{1}{\left(1+v_{1}^{\prime 2}\right)^{2}\left|v_{1}-v_{1}^{\prime}\right|^{2-\beta}} \\
& \leqq C\|\bar{g}\|_{3}\|\bar{f}\|_{s} \delta^{-2} .
\end{aligned}
$$

On the other hand, if $\left|v_{2}\right|<\eta\left|v_{1}\right|$ and $\left|v_{1}^{\prime}\right|<\delta\left|v_{1}\right|$, by the energy and momentum conservation we have

$$
v_{2}^{\prime 2}=v_{1}^{2}+v_{2}^{2}-v_{1}^{\prime 2}>\left(1-\delta^{2}\right) v_{1}^{2}
$$

and

$$
\left|v_{2}^{\prime}-v_{1}\right|<\left|v_{2}\right|+\left|v_{1}^{\prime}\right|<(\eta+\delta)\left|v_{1}\right| .
$$

so that $v_{2}^{\prime}$ is at most in a sphere centered in $v_{1}$ and with radius $(\eta+\delta)\left|v_{1}\right|$.

Its intersection with $E_{v_{1} v_{1}^{\prime}}$ has measure at most $\pi(\eta+\delta)^{2}\left|v_{1}\right|^{2}<4 \pi \eta^{2}\left|v_{1}\right|^{2}$. Therefore

$$
\begin{gathered}
J_{0}^{2}\left(\bar{g}_{i}, \bar{f}_{0}\right)\left(v_{1}\right)\left(1+v_{1}^{2}\right)^{s} \leqq C\|\bar{g}\|_{3}\|\bar{f}\|_{s}\left(1+v_{1}^{2}\right)^{s} \frac{\eta^{2} v_{1}^{2}}{\left[1+\left(1-\delta^{2}\right) v_{1}^{2}\right]^{s}} \\
\quad \cdot \int_{\mathbb{R}^{3}} d v_{1}^{\prime} \chi_{i}\left(v_{1}^{\prime}\right) \chi_{\delta}\left(v_{1}^{\prime}\right) \frac{1}{\left(1+v_{1}^{\prime 2}\right)^{3}\left|v_{1}-v_{1}^{\prime}\right|^{2-\beta}} \\
\leqq C \eta^{2}\left(1-\delta^{2}\right)^{-s}\left(1+\left|v_{1}\right|^{\beta}\right)\|\bar{g}\|_{3}\|\bar{f}\|_{s} .
\end{gathered}
$$


Finally, since

$$
\chi_{\delta}\left(v_{1}^{\prime}\right) \int_{E_{v_{1} v_{1}^{\prime}}} d \xi \chi_{\eta}^{c}\left(v_{2}^{\prime}\right) \frac{1}{\left(1+v_{2}^{\prime 2}\right)^{s}} \leqq \frac{C}{(s-1)\left(1+\bar{v}_{2}^{\prime 2}\right)^{s-1}},
$$

where $\bar{v}_{2}^{\prime 2}$ is the minimum value of $v_{2}^{\prime 2}$ compatible with the constraints, we have

and hence

$$
v_{2}^{\prime 2}=v_{1}^{2}+v_{2}^{2}-v_{1}^{\prime 2}>\left(1+\eta^{2}-\delta^{2}\right) v_{1}^{2}
$$

$$
J_{0}^{3}\left(\bar{g}_{i}, \bar{f}_{0}\right)\left(v_{1}\right)\left(1+v_{1}^{2}\right)^{s} \leqq C\|\bar{g}\|_{3}\|\bar{f}\|_{s}\left(1+\left|v_{1}\right|^{\beta}\right)(s-1)^{-1}\left(1+\eta^{2}-\delta^{2}\right)^{1-s},
$$

by the inequality (3.17).

In conclusion, collecting all the above estimates, we have:

$$
\begin{aligned}
& J_{0}\left(\bar{g}_{i}, \bar{f}_{0}\right)\left(v_{1}\right)\left(1+v_{1}^{2}\right)^{s} \leqq\|\bar{g}\|_{3}\|\bar{f}\|_{s}\left\{C \delta^{-2}+A\left(1+\left|v_{1}\right|^{\beta}\right) .\right. \\
& \left.\quad \cdot\left(\eta^{2}\left(1-\delta^{2}\right)^{-s}+(s-1)^{-1}\left(1+\eta^{2}-\delta^{2}\right)^{1-s}\right)\right\},
\end{aligned}
$$

for some $A>0$ not depending on $s$.

We choose $\eta=1 / \sqrt{s}, \delta=1 / s$ for which $\delta^{2} \leqq \eta^{2} / 2=1 / 2 s$. Therefore

$$
\left(1+\eta^{2}-\delta^{2}\right)^{1-s} \leqq\left(1+\eta^{2} / 2\right)^{1-s}=(1+1 / 2 s)^{1-s} \leqq C .
$$

On the other hand

$$
\eta^{2}\left(1-\delta^{2}\right)^{-s} \leqq \frac{1}{s\left(1-s^{-2}\right)^{s}} \leqq C s^{-1},
$$

so that (3.8) is proved with $\varepsilon(s)=C s^{-1}$.

\section{Proof of the Main Theorem}

Let $f_{0}(x, v),(x, v) \in T \times \mathbb{R}^{3}$ be positive and in $B_{l, s}$. Denote by $g_{0}$ its space average

$$
g_{0}(v)=\int_{T} d x f_{0}(x, v)
$$

and by $u_{0}=f_{0}-g_{0}$ its deviation. Let $g_{t}$ and $u_{t}$ be solutions of the initial value problems

$$
\partial_{t} g=Q(g, g), \quad g(0)=g_{0}
$$

and

$$
D_{t} u+u R g=\Phi_{t}:=Q(g, u)+J(u, g)+Q(u, u), \quad u(0)=u_{0} .
$$

Denote by

$$
f_{t}(x, v)=g_{t}(v)+u_{t}(x, v)
$$

then $f_{t}$ is a solution of the initial value problem for the Boltzmann equation (1.1). The initial value problem (4.2) can be uniquely solved for all $t$ if $s$ is large enough since $g_{0} \in B_{s}$ and the solution has the properties established in Sect. 2. Now we prove a local existence and uniqueness theorem for the problem (4.3). Let $\hat{u}_{t}(k, v), k \in 2 \pi \mathbb{Z}^{3}$ 
be the Fourier transform of $u_{t}(x, v)$. Consider the following integral equation in $B_{l, s}$ :

$$
\begin{aligned}
\hat{u}_{t}(k, v)= & \hat{u}_{0}(k, v) \exp -\left\{i k \cdot v t+\int_{0}^{t} d \tau\left(R g_{\tau}\right)(v)\right\} \\
& +\int_{0}^{t} d \tau \exp \left\{-\int_{\tau}^{t} d \sigma\left(i k \cdot v \sigma+R g_{\sigma}(v)\right)\right\} \hat{\Phi}_{\tau}(k, v),
\end{aligned}
$$

where $\hat{\Phi}_{\tau}(k, v)$ denotes the Fourier transform of $\Phi_{\tau}(x, v)$.

Proposition 4.1. There exists $s_{0}>0$ such that, for $s>s_{0}$ and $l>3 / 2$ if $u_{0} \in B_{l, s}$ and $g_{0} \in B_{s+\beta / 2}$, then any solution in $B_{l, s}$ of Eq. (4.5) satisfies the estimate:

$$
\left\|u_{t}\right\|_{l, s} \leqq\left\|u_{0}\right\|_{l, s} \exp [(2 C \ln 4) t]
$$

for some constant $C$ depending only on $s$ and $g_{0}$, provided that

$$
\left\|u_{0}\right\|_{l, s} \leqq(24 C)^{-1} \exp [-(2 C \ln 4) t] .
$$

Proof. Denote $Y(t)=\left\|u_{t}\right\|_{l, s}$ and $\bar{Y}(\tau)=\sup _{0 \leqq \sigma \leqq \tau} Y(\sigma)$.

By the estimates on the collision term in Proposition 3.1 and by Eq. (2.2), fixed $\varepsilon>0$, we can find $s_{0}$ such that

$$
\left|\Phi_{t}(\cdot, v)\right|_{l}\left(1+v^{2}\right)^{s} \leqq\left(A_{s}+\varepsilon|v|^{\beta}\right)\left\|u_{t}\right\|_{l, s}+A_{s}\left(1+|v|^{\beta}\right)\left(\left\|u_{t}\right\|_{l, s}\right)^{2}
$$

for some constant $A_{s}$ and $s>s_{0}$. Therefore, by Eq. (4.5), using (2.4), we have:

$$
Y(\sigma) \leqq Y(0)+\tau A_{s} \bar{Y}(\tau)+(\varepsilon / v) \bar{Y}(\tau)+\left(A_{s} / v\right) \bar{Y}(\tau)^{2} \text { for } \quad 0 \leqq \sigma \leqq \tau .
$$

Putting $C=\max \left(A_{s}+1, A_{s} / v\right)$, if $\varepsilon<v \tau$,

$$
0 \leqq Y(0)-(1-\tau C) \bar{Y}(\tau)+C \bar{Y}(\tau)^{2}
$$

Thus, for $\tau$ such that $\tau C=1 / 2$ we have, by continuity $Y$ as function of $\sigma$

$$
Y(\sigma) \leqq \bar{Y}(\tau) \leqq(1 / 4-\sqrt{1 / 16-Y(0) C}) / C \leqq 4 Y(0) \text { for } 0 \leqq \sigma \leqq \tau,
$$

if $Y(0) C<1 / 24$. In the last step we used the obvious inequality $\sqrt{1-16 Y(0) C}>$ $(1-8 Y(0) C / \sqrt{1-16 Y(0) C)}$. By iteration of the estimate (4.11) $n$ times, with $n=$ $[t / \tau]-1([x]=$ integer part of $x)$, we get (4.6) from (4.7).

Proposition 4.2. Given $s>s_{0}$, if $u_{0}$ satisfies (4.7) with $C$ large enough, and $g_{0} \in B_{s+\beta / 2}$, there exists a unique solution up to the time $t$, of Eq. (4.5), satisfying (4.6).

Proof. We define, by iteration

$$
\left(D_{t}+R g\right) u^{(n)}=\Phi^{(n-1)} n \geqq 1, \quad u^{(n)}(0)=u_{0}=u^{(0)},
$$

where

$$
\Phi^{(n)}=Q\left(g, u^{(n)}\right)+J\left(u^{(n)}, g\right)+Q\left(u^{(n)}, u^{(n)}\right) .
$$

For $n \geqq 2 \varphi^{(n)}:=u^{(n)}-u^{(n-1)}, \varphi^{(1)}:=u^{(1)}$ satisfies

$$
\left(D_{t}+R g\right) \varphi^{(n)}=F^{(n-1)},
$$


where

$$
\begin{aligned}
F^{(n-1)}= & \Phi^{(n-1)}-\Phi^{(n-2)}=Q\left(g, \varphi^{(n-1)}\right)+J\left(\varphi^{(n-1)}, g\right) \\
& +Q\left(u^{(n-1)}, \varphi^{(n-1)}\right)+Q\left(\varphi^{(n-1)}, u^{(n-2)}\right), \quad n \geqq 2, \\
F^{(0)}= & \Phi^{(0) .}
\end{aligned}
$$

By Proposition 3.1 we have

$$
\begin{aligned}
& \left|F^{(n-1)}(\cdot, v)\right|_{l}\left(1+v^{2}\right)^{s} \leqq\left(A_{s}+\varepsilon|v|^{\beta}\right)\left\|\varphi^{(n-1)}\right\|_{l, s} \\
& \quad+A_{s}\left(1+|v|^{\beta}\right)\left\{\left\|u^{(n-1)}\right\|_{l, s}+\left\|u^{(n-2)}\right\|_{l, s}\right\}\left\|\varphi^{(n-1)}\right\|_{l, s^{*}}
\end{aligned}
$$

Obviously the sequence $u^{(n)}$ satisfies the estimate (4.6) provided that (4.7) is satisfied. Therefore, proceeding as in Proposition 4.1, we get for $s>s_{0}$ :

where

$$
\bar{\varphi}_{\tau}^{(n)} \leqq \alpha \bar{\varphi}_{\tau}^{(n-1)}, \quad \alpha<1
$$

$$
\bar{\varphi}_{\tau}=\sup _{0 \leqq \sigma \leqq \tau}\left\|\varphi_{\sigma}\right\|_{l, s}
$$

provided that $\tau$ and $1 / C$ are small enough. Therefore, if $\varepsilon$ and $\tau$ are small enough the iteration converges for $\sigma \leqq \tau$. This procedure can be iterated to get the convergence up to time $t$ if $\left\|u_{0}\right\|_{l, s}$ is small. The uniqueness follows trivially.

Remark. Further regularity properties of the solution constructed above will be discussed later.

Now we consider the evolution of a perturbation of the equilibrium. We assume $f_{0}$ such that

$$
\int f_{0}(x, v) d x d v=1, \quad \int v f_{0}(x, v) d x d v=0, \quad \int v^{2} f_{0}(x, v) d x d v=3 .
$$

Then $f_{t}$ is expected to converge to $\omega$ defined in (2.6).

We write, for $t \geqq 0$,

We prove the following:

$$
f_{t}=\omega+u_{t}
$$

Proposition 4.3. Fixed $s_{0}$ and $l_{0}$ sufficiently large, for any $s>s_{0}$ and $l>l_{0}$ we can find $b, b^{\prime}, \gamma>0$ such that, if $\left\|u_{0}\right\|_{l, s}<b$, then there exists a unique solution of the Boltzmann equation in the strong sense of $B_{l-\varepsilon-1, s-(\varepsilon+1) / 2}$ for $\varepsilon>0$ and $u_{t}$ satisfies the bound

$$
\left\|u_{t}\right\|_{l, s} \leqq b^{\prime} \exp -\gamma t
$$

Proof. Let $h_{\alpha}(v) \alpha=1, \ldots, 5$ be defined as follows:

$$
h_{1}(v)=1, \quad h_{1+i}(v)=v_{i} i=1, \ldots, 3, \quad h_{5}(v)=(\sqrt{6})^{-1}\left(v^{2}-3\right),
$$

and let

$$
\psi_{\alpha}=h_{\alpha} \sqrt{\omega}, \quad \alpha=1, \ldots, 5 .
$$

$\left\{\psi_{\alpha}\right\}_{\alpha=1, \ldots, 5}$ are in $L_{2}\left(\mathbb{R}^{3}, H_{l}(T)\right)$ and are orthonormal with respect to the scalar product

$$
(f, g)=\int_{\mathbb{R}^{3}} d v \sum_{k \in 2 \pi \mathbb{Z}^{3}} \hat{f}^{*}(k, v) \hat{g}(k, v)\left(1+k^{2}\right)^{l} .
$$


Let $P$ be the projector on the subspace spanned by $\left\{\psi_{\alpha}\right\}$ :

$$
P f=\sum_{\alpha=1,5}\left(f, \psi_{\alpha}\right) \psi_{\alpha} .
$$

Now we decompose $u_{0}$ as follows: $u_{0}=u_{0}^{(1)}+u_{0}^{(2)}+u_{0}^{(3)}$ with

$$
\begin{aligned}
& u_{0}^{(1)}(x, v)=\sqrt{\omega}(1-P)\left[\sqrt{\omega}^{-1} \chi_{M}(v) u_{0}(x, v)\right], \\
& u_{0}^{(2)}(x, v)=\chi_{M}^{c}(v) u_{0}(x, v), \\
& u_{0}^{(3)}(x, v)=\sqrt{\omega} P\left[\sqrt{\omega}^{-1} \chi_{M}(v) u_{0}(x, v)\right],
\end{aligned}
$$

where, for $M>0, \chi_{M}(v)=\left\{\begin{array}{l}1 \text { if }|v|<M \\ 0 \text { otherwise }\end{array}\right.$, and $\chi_{M}^{c}=1-\chi_{M}$.

Furthermore we put

$$
f_{0}^{(i)}=u_{0}^{(i)} \sqrt{\omega}^{-1}, \quad i=1,2,3 .
$$

Since $u_{0} \in B_{l, s}, f_{0}^{(1)}$ and $f_{0}^{(3)}$ are in $L_{2}\left(\mathbb{R}^{3}, H_{l}(T)\right)$.

\section{Putting}

$$
\begin{aligned}
L f & =-(Q(\omega, f)+Q(f, \omega)), \\
\tilde{L} f & =\sqrt{\omega^{-1}} L(\sqrt{\omega} f), \\
v f & =f R \omega, \\
K f & =v f-L f,
\end{aligned}
$$

consider the linear initial value problems, for $z_{i} \in L_{\infty}\left([0,+\infty), B_{l, s}\right), i=1,2,3$ :

$$
\begin{aligned}
& \left(D_{t}+v\right) u_{2}=\chi_{M}^{c} K u_{2}+\left[Q\left(z_{1}, z_{2}\right)+Q\left(z_{2}, z_{3}\right)\right], \\
& \left(D_{t}+\tilde{L}\right) f_{1}=(1-P) \sqrt{\omega}^{-1} \chi_{M} K u_{2}, \\
& \left(D_{t}+\tilde{L}\right) f_{3}=P \sqrt{\omega}-1 \chi_{M} K u_{2}, \\
& \quad u_{2}(0)=u_{0}^{(2)}, \quad f_{1}(0)=f_{0}^{(1)}, \quad f_{3}(0)=f_{0}^{(3)} .
\end{aligned}
$$

We put $u^{(i)}(t)=\sqrt{\omega} f^{(i)}(t)$ and $u(t)=u_{1}(t)+u_{2}(t)+u_{3}(t)$. For $z_{1}=z_{2}=u$ and $z_{3}=0$, $\omega+u(t)$ is a solution of the Boltzmann equation.

We begin by studying Eq. (4.30) $)_{1}$ in integral form (the same as (4.5) with $g$ replaced by $\omega$ and $\phi$ replaced by right-handside of $(4.30)_{1}$.

Lemma 1. There is $\bar{v}>0$ such that $v(v) \geqq \bar{v}\left(1+|v|^{\beta}\right)$.

Proof. See Grad [3] or alternatively Proposition 2.2.

We put:

$$
\|f\|_{l, s, \gamma}=\sup _{t \geqq 0} e^{\gamma t}\|f(t)\|_{l, s^{*}}
$$

Lemma 2: For $s$ and $l$ sufficiently large, there are $C, \bar{C}$ such that any solution of $(4.30)_{1}$ satisfies

$$
\begin{aligned}
\left\|u_{2}\right\|_{l, s \gamma} \leqq & \left\|u_{0}\right\|_{l, s}+C\left\|z_{2}\right\|_{l, s, \gamma}\left(\left\|z_{1}\right\|_{l, s, \gamma}+\left\|z_{3}\right\|_{l, s, \gamma}\right) \\
& +2 \bar{v}^{-1}\left[\varepsilon(s)+C\left(1+M^{\beta}\right)^{-1}\right]\left\|u_{2}\right\|_{l, s, \gamma}
\end{aligned}
$$

for $0<\gamma<v_{0} / 2=1 / 2 \inf v(v)$. 
Proof. We have

$$
\begin{aligned}
\hat{u}_{2}(k, v, t)= & e^{-\{(i k \cdot v+v(v)) t\}} \hat{u}_{0}^{(2)}(k, v)+\int_{0}^{t} d \tau e^{-(i k \cdot v+v(v))(t-\tau)} \\
& \cdot\left[\chi_{M}^{c} K u_{2}(\tau)+Q\left(z_{1}(\tau), z_{2}(\tau)\right)+Q\left(z_{2}(\tau), z_{3}(\tau)\right)\right] \hat{(}(k, v) .
\end{aligned}
$$

By (3.8) and Lemma 1

$$
\begin{aligned}
& \left(1+v^{2}\right)^{s}\left(\sum_{k \in 2 \pi \mathbb{Z}^{3}}\left(1+k^{2}\right)^{l} \int_{0}^{t} \mid d \tau e^{-(i k \cdot v+v(v))(t-\tau)} \chi_{M}^{c}\left(\left.K u_{2}(\tau) \hat{)}(k, v)\right|^{2}\right)^{1 / 2}\right. \\
& \leqq 2 \bar{v}^{-1}\left(C(s)\left(1+M^{\beta}\right)^{-1}+\varepsilon(s)\right)\left\|u_{2}\right\|_{l, s, \gamma} e^{-\gamma t} .
\end{aligned}
$$

On the other hand by (3.7),

$$
\left(1+v^{2}\right)^{s}\left|Q\left(z_{1}(\tau), z_{2}(\tau)\right)\right|_{l} \leqq C\left(1+|v|^{\beta}\right)\left\|z_{1}\right\|_{l, s, \gamma}\left\|z_{2}\right\|_{l, s, \gamma} e^{-2 \gamma \tau}
$$

and therefore by Lemma 1

$$
\begin{aligned}
& \left\|\int_{0}^{t} d s e^{-(i k \cdot v+v(v))(t-\tau)} Q\left(z_{1}(\tau), z_{2}(\tau)\right)\right\|_{l, s} \\
& \quad \leqq C\left\|z_{1}\right\|_{l, s, \gamma}\left\|z_{2}\right\|_{l, s, \gamma} \sup _{v \in \mathbb{R}^{3}} \int_{0}^{t} d \tau\left(1+|v|^{\beta}\right) e^{-2 \gamma \tau} e^{-v(v)(t-\tau)} \\
& \quad \leqq 2 \bar{v}^{-1} C\left\|z_{1}\right\|_{l, s, \gamma}\left\|z_{2}\right\|_{l, s, \gamma} e^{-\gamma t} .
\end{aligned}
$$

Lemma 3. Suppose $M$, l, s large enough and $\left\|z_{i}\right\|_{l, s, \gamma}<+\infty, i=1,2,3$. There exists a unique solution of Eq. (4.33), $u_{2} \in L_{\infty}\left([0,+\infty), B_{l, s}\right) \cap C_{0}\left([0,+\infty), B_{l-\varepsilon, s-\varepsilon / 2}\right)$ for any $\varepsilon>0$. Suppose in addition that $z_{i} \in C_{0}\left([0,+\infty), B_{l-\varepsilon, s-\varepsilon / 2}\right), i=1,2,3$ for some $\varepsilon>0$. Then $u_{2} \in C_{1}\left([0,+\infty), B_{l-\varepsilon-1, s-(\varepsilon+1) / 2}\right)$ and satisfies Eq. (4.30) $)_{1}$ strongly in $B_{l-\varepsilon-1, s-(\varepsilon+1) / 2}$.

Proof. Existence and uniqueness follow easily by iteration and estimate (4.32). Since $Q\left(z_{1}, z_{2}\right), Q\left(z_{2}, z_{3}\right)$ and $K u_{2} \in L_{\infty}\left([0,+\infty), B_{l, s-\beta / 2}\right)$, then, by Eq. (4.33) $u_{2} \in C_{0}\left([0,+\infty), B_{l-\varepsilon, s-\varepsilon / 2}\right)$.

Moreover, putting

$$
\Psi=\left(-v \cdot \nabla_{x}-v+\chi_{M}^{c} K\right) u_{2}+\left[Q\left(z_{1}, z_{2}\right)+Q\left(z_{2}, z_{3}\right)\right]
$$

we get $\Psi \in C_{0}\left([0,+\infty), B_{l-\varepsilon-1, s-(\varepsilon+1) / 2}\right)$ provided that $z_{i} \in C_{0}\left([0,+\infty), B_{l-\varepsilon, s-\varepsilon / 2}\right)$, $i=1,2,3$. The convergence of $h^{-1}\left(u_{2}(t+h)-u_{2}(t)\right)$ to $\Psi(t)$ in $B_{l-\varepsilon-1, s-(\varepsilon+1) / 2}$, for $h \rightarrow 0$, is standard.

The following Proposition summarizes the properties of the operator $\tilde{L}$ we use in the proof:

Proposition 4.4. Consider the initial value problem

$$
\left(D_{t}+\tilde{L}\right) w(t)=\phi(t), \quad w(0)=w_{0} .
$$

Suppose $w_{0} \in B_{l, s}$ and $\phi \in L_{\infty}\left([0,+\infty), B_{l, s}\right) \cap C_{0}\left([0,+\infty), B_{l-\varepsilon-1, s-\varepsilon / 2-1 / 2}\right)$ for $\varepsilon>0$ and some $l$ and $s$ sufficiently large. Then Eq. (4.38) has a unique solution $w(t) \in B_{l, s}$ for $t \geqq 0$ which is strongly differentiable in $B_{l-\varepsilon-1, s-(\varepsilon+1) / 2}$. Moreover if 
$\phi(t) \in \operatorname{Ker} P$ for $t \in[0,+\infty)$, and $w_{0} \in \operatorname{Ker} P$, then $w(t) \in \operatorname{Ker} P$ and there are positive constants $\mu, d, d^{\prime}$ such that for $0<\gamma<\mu$,

$$
\|w\|_{l, s, \gamma} \leqq d\left\|w_{0}\right\|_{l, s}+d^{\prime}\|\phi\|_{l, s, \gamma}
$$

Proof. Proposition 4.4 follows easily by ref. [2], Lemma 4.4 .

Given $u_{2}$ as in Lemma 3 with $z_{1}=z_{2}$ and $z_{3}=0$, we next consider the solution of $(4.30)_{2}$ and $(4.30)_{3}$ as given by Proposition 4.4 .

Lemma 4. Suppose $u(t)=u_{1}(t)+u_{2}(t)+u_{3}(t) \in B_{l, s} \forall t>0$, for $s>3$. Then $\left(h_{\alpha}, u(t)\right)$ $=0, \alpha=1, \ldots, 5$.

Proof. The scalar products make sense since $s>3$. Furthermore the scalar products $\left(h_{\alpha}, Q(\omega, u(t))\right)$ and $\left(h_{\alpha}, Q(u(t), u(t))\right)$ make sense by the estimates on the collision operators and vanish by direct computation. Therefore $\left(h_{\alpha}, u(t)\right)=0 \operatorname{since}\left(h_{\alpha}, u_{0}\right)=0$.

We have, for any $f \in B_{l, s}, l>1, s>1 / 2$,

$$
\left(v \cdot \nabla_{x}+\tilde{L}\right) P f=0=P\left(v \cdot \nabla_{x}+\tilde{L}\right) f
$$

by the same arguments as in the proof of Lemma 4 .

Therefore, by Proposition 4.4, $P f_{1}(t)=0, \forall t>0$, and, by Lemma 4

$$
P f_{2}(t)=-P f_{3}(t) \forall t>0 \text {. }
$$

Lemma 5. There is a constant $C$ such that

$$
\left\|u_{3}(t)\right\|_{l, s} \leqq C\left\|u_{2}(t)\right\|_{l, s}
$$

Proof. $f_{3}(t)=P f_{3}(t)$ by Eq. (4.40) and Proposition 4.4, since $(1-P) f_{3}(0)=0$. Therefore

$$
\begin{aligned}
\left\|u_{3}(t)\right\|_{l, s} & =\left\|\sqrt{\omega} f_{3}\right\|_{l, s}=\left\|\sqrt{\omega} P f_{2}\right\|_{l, s} \leqq\left\|P f_{2}\right\|_{l, s} \\
& \leqq \sum_{\alpha=1,5}\left(\sqrt{\omega}-1 u_{2}, \psi_{\alpha}\right) \sup _{v \in \mathbb{R}^{3}}\left[\left(1+v^{2}\right)^{s}\left|\psi_{\alpha}(v)\right|\right] \\
& \leqq C_{s} \sum_{\alpha=1,5} \int d x d v\left|u_{2}(x, v)\right| h_{\alpha}(v) \\
& \leqq C_{s}\left\|u_{2}(t)\right\|_{l, s} \int d v h_{\alpha}(v)\left(1+v^{2}\right)^{-s} \leqq C\left\|u_{2}(t)\right\|_{l, s} \text { for } s>3 .
\end{aligned}
$$

Lemma 6. For l, s as in Proposition 4.4 there is a constant $C_{M}$ such that, for $0<\gamma<\mu$,

$$
\left\|u_{1}\right\|_{l, s, \gamma}<C_{M}\left(\left\|u_{2}\right\|_{l, s, \gamma}+\left\|u_{0}\right\|_{l, s}\right) \text {. }
$$

Proof. Using Proposition 4.4, with $w_{0}=f_{0}^{(1)}$ and $\phi(t)=(1-P) \sqrt{\omega}^{-1} \chi_{M} K u_{2}$, we have, since $f_{0}^{(1)}$ and $(1-P) \sqrt{\omega}^{-1} \chi_{M} K u_{2}$ are in $\operatorname{Ker} P$,

$$
\left\|f_{1}\right\|_{l, s, \gamma} \leqq d\left\|f_{0}^{(1)}\right\|_{l, s}+d^{\prime}\left\|(1-P) \sqrt{\omega}^{-1} \chi_{M} K u_{2}\right\|_{l, s, \gamma}
$$

By (3.7) and the arguments used in the proof of Lemma 5 we have

Moreover

$$
\left\|(1-P) \sqrt{\omega}^{-1} \chi_{M} K u_{2}\right\|_{l, s, \gamma} \leqq C_{M}\left\|u_{2}\right\|_{l, s, \gamma}
$$

$$
\left\|f_{0}^{(1)}\right\|_{l, s}=\left\|(1-P) \sqrt{\omega}^{-1} \chi_{M} u_{0}\right\|_{l, s} \leqq C_{M}\left\|u_{0}\right\|_{l, s^{*}}
$$


Now we can prove Proposition 4.3. From now on we fix $s$ and $M$ such that $2 \bar{v}^{-1}\left(\bar{C}\left(1+M^{\beta}\right)^{-1}+\varepsilon(s)\right)<1 / 2$. By Lemma 2 :

$$
\left\|u_{2}\right\|_{l, s, \gamma} \leqq 2\left\|u_{0}\right\|_{l, s}+2 C\left\|z_{2}\right\|_{l, s, \gamma}\left(\left\|z_{1}\right\|_{l, s, \gamma}+\left\|z_{3}\right\|_{l, s, \gamma}\right)
$$

By Lemma 5, Lemma 6 and inequality (4.48) we see that for $l>l_{0}, s>s_{0}$ and $0<\gamma$ $<\min \left(\mu, \bar{v}_{0} / 2\right),\|u\|_{l, s, \gamma}$ must satisfy the inequality:

$$
\|u\|_{l, s, \gamma} \leqq C_{1}\left\|u_{0}\right\|_{l, s}+C_{2}\left\|z_{2}\right\|_{l, s, \gamma}\left(\left\|z_{1}\right\|_{l, s, \gamma}+\left\|z_{3}\right\|_{l, s, \gamma}\right)
$$

(here $C_{1}$ and $C_{2}$ depend only on the choice of $M$ and $s$ ).

Now we consider the following iterative procedure:

$$
\begin{gathered}
\left(D_{t}+L\right) u^{(n)}=Q\left(u^{(n-1)}, u^{(n-1)}\right), \quad n \geqq 1, \\
u^{(0)}=0, \quad u^{(n)}(0)=u_{0}, \quad n \geqq 1 .
\end{gathered}
$$

By virtue of the estimate (4.49) (with $z_{3}=0, z_{1}=z_{2}=u^{(n-1)}$ ) we easily obtain

$$
\left\|u^{(n)}\right\|_{l, s, \gamma}<\left(1-\sqrt{1-4 C_{1} C_{2}\left\|u_{0}\right\|_{l, s}}\right)\left(2 C_{2}\right)^{-1}:=C^{\prime}
$$

provided that $\left\|u_{0}\right\|_{l, s}<\left(4 C_{1} C_{2}\right)^{-1}$. Moreover, again by (4.49) (with $z_{1}=u^{(n-1)}$, $z_{2}=u^{(n-1)}-u^{(n-2)}$ and $\left.z_{3}=u^{(n-2)}\right)$,

$$
\left\|u^{(n)}-u^{(n-1)}\right\|_{l, s, \gamma} \leqq \alpha\left\|u^{(n-1)}-u^{(n-2)}\right\|_{l, s, \gamma}
$$

with $\alpha<1$ if $\left\|u_{0}\right\|_{l, s}$ is sufficiently small (by (4.51)).

Denoting by $u$ limit of $u^{(n)}$, it is not hard to prove that it satisfies:

$$
\hat{u}(k, v, t)=e^{-(i k \cdot v+v(v)) t} \hat{u}_{0}(k, v)+\int_{0}^{t} d \tau e^{-(i k \cdot v+v(v))(t-\tau)}[K u(\tau)+(Q(u(\tau), u(\tau)))]^{\hat{\prime}}(k, v) .
$$

The strong differentiability of $u$ in $B_{l-\varepsilon-1, s-\varepsilon-1 / 2}$ follows by the same arguments as in Lemma 3.

Proof of Theorem 1. We fix $s$ and $l$ according to the previous lemmas and assume $f_{0} \in B_{l, s+1}, g_{0}$ to be the space average of $f_{0}$ and $u_{0}$ its deviation. By Prop. $2.3 g_{t} \in B_{s+1}$ and approaches the equilibrium in $B_{s}$. Therefore there is $t^{*}>0$ such that

$$
\left\|g_{t}-\omega\right\|_{s}<b / 2 \quad \forall t \geqq t^{*}
$$

where $b$ is the one introduced in Proposition 4.3. By Proposition $4.1\left\|u\left(t^{*}\right)\right\|_{l, s} \leqq b / 2$ if $\left\|u_{0}\right\|_{l, s}<a$, where $a$ is fixed by (4.6) and (4.7). Therefore at time $t^{*}$ we have

$$
\left\|f_{t^{*}}-\omega\right\|_{l, s} \leqq\left\|f_{t^{*}}-g_{t^{*}}\right\|_{l, s}+\left\|g_{t^{*}}-\omega\right\|_{s}<b .
$$

Using Prop. 4.3 with initial time $t^{*}$ and initial datum $f_{t}-\omega$, it follows that $f_{t}$ exists uniquely also for $t>t^{*}$ and converges to $\omega$ for $t \rightarrow \infty$. By the same arguments as in Lemma $3 f \in C^{1}\left((0, \infty), B_{l-\varepsilon-1, s-(\varepsilon+1) / 2}\right)$. Therefore Eq. (1.1) can be understood in $B_{l-\varepsilon-1, s-(\varepsilon+1) / 2}$ for all $t>0$.

Remark 1. Since (4.54) follows by the $H$-theorem, $t^{*}$ is not explicitly known and then we have no control on the rate of convergence of the solution to equilibrium. 
Remark 2. By the same arguments of Sect. 4, it also follows that the solution constructed in Theorem 1 is stable in $B_{l, s}$. Namely, the constant $\mu$ of Proposition 4.4 is locally uniformly (with respect to $\omega$ ) bounded away from zero, as can be seen by a slight elaboration of the argument in [2]. It then follows from the proof of Proposition 4.3, that its constant $s_{0}, l_{0}, b, b^{\prime}, \gamma$ can be chosen locally independent of $\omega$, and that we can take $b^{\prime} \rightarrow 0$, when $b \rightarrow 0$. Therefore, analogously to the proof of Theorem 1, we can conclude that the solutions constructed in Theorem 1 are stable in $B_{l, s}$, i.e. given $\varepsilon>0$, and $f_{0}$ as in Theorem 1 , there is a $\delta>0$ such that

$$
\left\|f_{t}-\tilde{f}_{t}\right\|_{l, s}<\varepsilon, \quad t>0, \text { if } \tilde{f}_{0} \in B_{l, s} \text { and }\left\|f_{0}-\tilde{f}_{0}\right\|_{l, s}<\delta .
$$

In particular the space homogeneous Boltzmann Equation is stable in this sense also when the initial value is far from equilibrium.

\section{References}

1. Carleman, T. Problèms mathématiques dans la théorie cinétique des gaz Uppsala: Almquist \& Wiksells 1957

2. Ukai, S.: Proc. Jpn. Acad. 50, 179-184 (1975)

3. Grad, H.: Proc. Symp. in Appl. Math. 17, 154-183 (1965), Rarified Gas Dynamics, I, 26-59 (1963)

4. Illner, R., Shinbrot, M.: Commun. Math. Phys. 95, 217-226 (1984)

5. Arkeryd, L.: Arch. Rat. Mech. Anal. 86, 85-97 (1984); Commun. Math. Phys. 105, (1986)

6. Truesdell, C., Muncaster, R. G.: Fundamentals of Maxwell's kinetic theory of a simple monoatomic gas, New York: Academic Press 1980

7. Arkeryd, L.: J. Stat. Phys. 31, 347-361 (1983)

8. Maslova, N. B., Tchubenko, R. P.: Dokl. Akad. Nauk. SSSR 202, 800-803 (1972). Vestn. Leningr. Univ. 1, 100-105 (1973). 7, 109-113 (1976). Vestn. Leningr. Univ. 13, 90-97 (1976)

Communicated by J. L. Lebowitz

Received September 11, 1986; in revised form January 13, 1987 
